\title{
Sexual health in general practice: history and the partner history
}

\author{
Philippa Matthews
}

General Practitioner, Killick Street Health Centre, London, UK

Correspondence to Dr Philippa Matthews, Killick Street Health Centre, 75 Killick Street, Islington, London N1 9RH, UK; philippa.matthews@nhs.net

\section{Mad Men}

The 50th anniversary of the contraceptive pill has been much discussed, and women of the generation that obtained 'the pill' in its earliest days have been telling how they had to pretend they were married or had a fiancé.

A brief fictionalised account of this era was given in one of the earliest episodes of the nuanced television series, Mad Men, set at around this time in New York. The single Peggy has been tipped off by her colleague Joan about a gynaecologist who will give her contraception. We find Peggy in a hospital gown sitting on the doctor's couch, studying an information booklet entitled 'It's Your Wedding Night'. The cover has a picture of a bride in full regalia, carried in the arms of her groom.

The doctor enters the room, lights up a cigarette and puffs. "I see from your chart - and your finger - that you're not married." "That's right", responds the nervous Peggy. Her doctor recommences in a way that gives us some hope for Peggy: "I'm not here to judge you - nothing wrong with a woman being practical about the possibility of sexual activity. Although as a doctor I would like to think that putting a woman into this position will not turn her into some kind of a strumpet". Hopes dashed - and by this time he is inserting a speculum. "I will warn you now that I will take you off this medicine if you abuse it. It's for your own good really. The fact is, even in our modern times, easy women don't find husbands." Peggy assures him she is responsible. He gives her the prescription, saying: "They are eleven dollars a month, so don't think you have to go out to become the town pump just to get your money's worth. Excuse my French".

\section{Swinging 60s}

It is apparent that in the UK women faced similar, often worse, experiences, and I heard from one family planning clinic doctor that the clinic staff would, after providing contraception to a woman, investigate whether she had actually got married when she had said she would.

So one can quite imagine, as the 1960s and 1970s rolled on, the effect this legacy might have on those pioneer doctors and nurses who were trying to give women control over their reproductive health. I can imagine there would be a strong and natural inclination not to ask a woman about her partner - or lack of partner. None of their business, judgemental, irrelevant. Many of our teachers and role models might, quite justifiably, have actively avoided this area of questioning.

This avoidance of taking a partner history might have been compounded by the historical, and profound, separation of 'venereal disease (VD)' clinics from 'family planning' clinics, despite the fact were both were working to minimise the unwanted consequences of sex. Clinics to tackle and treat sexually transmitted infections (STIs) were established partly in response to the needs of soldiers returning from World War I. In the publicity and information the men were, to a degree, portrayed as the innocent victims - of the other service users: female prostitutes. By the time of World War II, 'manly' men were being encouraged to use protection. Efforts to control infection through partner notification were already being made (although there were hopes that peace time would render this unnecessary). In other words, these services had recognised a clinical need to take partner histories.

The attendees of genitourinary (GUM) clinics, and indeed the specialty as a whole, remained seriously stigmatised for many years, many would say decades, after World War II. In the 1940s and 1950 s, when barrier contraceptive methods, such as cervical caps, were beginning to be available to women, it would have been inconceivable to link together these 
two spheres of sexual health. Contraception was for nice, married women - and clearly nothing to do with STIs. Oral contraception arrived in this environment.

Sexual behaviour changed, and herpes and genital warts were becoming a great deal more common through the 1960s and 1970s. But perhaps many doctors and nurses providing contraceptive services were deskilled - almost deliberately deskilled - in sexual history taking? The clinical value was not appreciated in the context of the provision of contraceptive care. And still, perhaps, it sounded altogether too much like prying, too much as though a woman's marital status might be being determined?

\section{Why ask?}

In fact there is great clinical value in taking a partner history as part of a full sexual history. Large studies tell us that those who have had a change of sexual partner in the last 3 months, or those who have had two or more partners in the last 12 months, are at higher risk of having chlamydia (though with any given individual one must not be too literal in interpreting what are rules of thumb). The gender or country of origin of the sexual partner(s) may help us weigh up risk of HIV, and we may need to consider the partner history quite far back if we are worried about a late presentation of HIV. We may find that the patient is in a mutually first, mutually monogamous relationship - and at no apparent risk of STIs. And, as we ask and listen, we may learn things about the quality of the relationship that are important to our patient - their relief as they confide their fears that their partner might be having sex with other people, and should they have tests for infections? Or we may become concerned if a young person reveals the much greater age of their partner. If a diagnosis of an STI has been made we may act on the partner history to explain the need for partner notification and treatment (if the STI is one for which this is indicated: this is not necessarily the case for some STIs such as genital warts or herpes).

Being able to take a partner history (indeed a full sexual history - other aspects will be covered in future articles in this series) is a key skill of the modern 'family' doctor. The value of taking partner histories becomes more apparent the more you ask, and, once strategies have been developed, it takes very little time.

\section{What to ask}

So which questions work? A 24-year-old newly registered patient tells me she has "Just come for some more pills". We have personal lists in our practice - so I will take pride in using this very first consultation to get to know her - just a little - and start to build our relationship. Has she just moved? What brought her to the area? Is she working? Is she living alone? How is she finding life in London?

If I have asked a few 'social' questions to get to know her and her personal circumstances; I might already have been told that she has a partner, but, if not, I cannot assume that she has one simply because she is on the pill. It is interesting how many women choose to carry on with the pill even when they have no contraceptive need, as a 'just in case' or because periods were heavier or more painful without it. I look after women who have lived in, or are from, an array of other countries. Sometimes they have been told the pill is indicated for a reason we would not necessarily recognise, for example, "I had an ovarian cyst that went after I was given the pill".

I find the GUM traditional opening question for the sexual history "When did you last have sex?" or "When did you last have sex with someone who wasn't your regular partner?" a little too bald for my clinical context. I prefer "Do you have a partner at present?" "How long have you been together?" The questions so far from a 'family doctor' will probably feel quite natural for a patient. In fact they are almost 'safe' social questions. However, if I want to move on to take a more detailed partner history I think I need to explain to my patient why, I need to reveal my agenda. So I might say: "We find quite a few under-25s have sexually transmitted infections, have you ever had a test for chlamydia? Do you think you could be at risk? Could I ask you some questions to check?” Or I might say: "We find sexually transmissible infections to be quite common, so we are trying to talk to our patients using contraception to see if they could be at risk". Whatever my motivation for taking a sexual history, I will want my patient to understand it - as was discussed in the preceding article in this series. Then (if she has been with her current partner more than a few weeks) I will return to the partner history. "You said you have been with your boyfriend 18 months - have you had sex with anyone else in that time?" I can then ask the mirror question: "Has your partner had sex with anyone else in that time?" This is one of the most useful questions to ask, because I will then gain insight into the woman's own sense of risk. "Well you know what men are like" frees me to discuss STIs and offer testing, whereas a calm and confident "No" means I will assess risk on the basis of other aspects of the history and clinical picture.

I will explore the partner history back to the most recent risk. If she is not currently in a sexual relationship I will find out when she last had sex or if she has had a sexual partner. This short sequence of questions making up a partner history brings helpful information surprisingly fast - whether the patient is at reasonably high risk, because they had a partner change quite recently, or whether they have been in an apparently mutually monogamous marriage for 9 years: one can establish the situation quickly. How far back I take the history, and what other questions I might ask, will be influenced by my original motivation for taking the history - the patient with possible HIV-related symptoms might be asked: "Have you ever had sex 
with someone from a country where HIV is common?" "Have you ever injected drugs?" "Have you ever had an HIV test?" alongside other aspects of the sexual history, such as assessing condom use and asking about sexual practices.

\section{What to believe?}

Providing a confidential service - and being seen to provide it - are essential components to helping patients feel able to talk. This is going to be even more important if your practice serves a small and stable community. But even if we provide a most secure space for talking, how do I know if the patient is lying to me? And anyway, how would someone know if their partner was secretly having sex with other people? I don't; they may not. The approach I take is invariable: I believe what the patient has told me. This works, treats the patient with respect and avoids causing offence. I find that if the patient has understood why I am asking my questions (i.e. why it is relevant to their health) then they will also understand the implications if some of the information is wrong. If the patient is asymptomatic, and I have found no evidence of risk of STI, I will leave it there: "From what you tell me you are not at risk of having an STI, but I am quite happy to do tests for chlamydia or HIV anyway if you would like these". Alternatively I might have been taking a sexual history because a patient has symptoms which might, or might not, be caused by an STI - a young man with troublesome mouth ulcers, or a 40-year-old woman with intermenstrual bleeding. I might need to exclude HIV or chlamydia, respectively, but non-STI causes might be much more likely. So finding a 'no apparent risk' sexual history I might say: "From what you tell me you are not at risk of having [HIV, chlamydia]. Would you like me to do a test to rule that option out altogether, or shall we look into other possible causes first?"

The 'no apparent risk' group are important to us in general practice. If a GUM clinic attender is at no apparent risk, it simply begs the question: "Why are they there then? Surely they must be?" "Everyone is at risk of an STI" makes perfect sense from a GUM perspective - there is no point in assessing risk, except to identify those at highest risk who might need more detailed health promotion advice. In general practice this is not the case. Sexual histories taken frequently, normalised, in the general practitioner (GP) context reveal there really are a lot of people at no, or very low, risk of having an STI. In fact our service users populate the entire spectrum of sexual health risk, with plenty at the high end too.

Once he had established Peggy had the 'wrong' marital status, Peggy's doctor took a 'No questions asked' (but lecture nonetheless delivered) approach. We know nowadays that lecturing is not a strategy for health promotion (let alone the imposition of moral values!). We should also have confidence that a 'No questions asked' approach belongs to times past.

If you would like to learn more about partner history-taking in general practice see e-GP on http:// www.e-GP.org.uk - sessions 11_001 through 11_003.

\section{Future articles}

This series of articles will explore a variety of the practical aspects of providing sexual health care as a GP or practice nurse.

\section{Competing interests None.}

Provenance and peer review Commissioned; internally peer reviewed. 